\title{
Del estallido a la lucha de clases. Claves para el análisis de los resultados del plebiscito por una nueva Constitución en Chile
}

\section{From the uprising to class struggle. Keys for the analysis of the results of the plebiscite for a new constitution in Chile}

\author{
Álvaro Arancibia-Bustos ${ }^{1}$ \\ Universidad Alberto Hurtado. Santiago de Chile, Chile \\ alvz.arancibia@gmail.com \\ ORCID: https://orcid.org/0000-0003-1565-4579 \\ José Flores-Capriles ${ }^{2}$ \\ Universidad Alberto Hurtado. Santiago de Chile, Chile \\ florescapriles.jose@gmail.com \\ ORCID: https://orcid.org/0000-0002-1434-6219

\section{Susana Gutiérrez-Peñaloza ${ }^{3}$} \\ Universidad Alberto Hurtado. Santiago de Chile, Chile \\ susanagp95@gmail.com \\ ORCID: https://orcid.org/0000-0001-5746-6766
}

Citar como: Arancibia-Bustos, Á., Flores-Capriles, J. y Gutiérrez-Peñaloza, S. (2021). Del estallido a la lucha de clases. Claves para el análisis de los resultados del plebiscito por una nueva Constitución en Chile. Desde el Sur, 13(1), e004

\section{RESUMEN}

El presente artículo tiene por objetivo indagar la relación entre posición de clase y voto en el plebiscito por una nueva Constitución en Chile. Para lo anterior, se tomaron los resultados de voto a nivel comunal publicados por el SERVEL y se asociaron estadísticamente, a partir del coeficiente de correlación de Pearson, con posiciones de clase considerando el esquema de explotaciones múltiples de Erik Olin Wright. Los resultados muestran una alta correlación positiva entre la opción «apruebo», la opción «Convención Constitucional», y el proletariado; en tanto, esta relación se invierte, con una alta fuerza negativa, en

\footnotetext{
1 Sociólogo de la Universidad de Chile. Estudiante del magíster y doctorado en Sociología de la Universidad Alberto Hurtado.

2 Licenciado en Sociología de la Universidad Alberto Hurtado.

3 Estudiante de Sociología de la Universidad Alberto Hurtado.
} 
el caso de la burguesía y los expertos directivos. Estos resultados sugieren una polarización en la defensa de los intereses de clase, a partir de la posibilidad de modificar el statu quo consagrado por la actual Constitución.

\section{PALABRAS CLAVE}

Estallido social, Constitución, plebiscito, clases sociales, conciencia de clase

\section{ABSTRACT}

The aim of this article is to investigate the relationship between class and voting in the plebiscite for a new constitution in Chile. To this end, the results of the vote were taken and statistically associated with class. The results show a high positive correlation between the Approve option, the Constitutional Convention option, and the proletariat. At the same time, this relationship is reversed, with a high negative correlation, in the case of the bourgeoisie and decision makers. These results suggest a polarization in the defense of class interests, regarding the prospect of modifying the status quo enshrined in the current constitution.

\section{KEYWORDS}

Social uprising, constitution, plebiscite, social classes, class consciousness

\section{Introducción}

El 18 de octubre, como efecto del alza de la tarifa en el transporte de Santiago, se convirtió en una fecha clave para importantes cambios estructurales en Chile. Las protestas realizadas a lo largo del estallido social reflejaban una crisis latente que apuntaban al alto costo de la vida en el país y la alta brecha de desigualdad que se han mantenido gracias a las políticas neoliberales de los últimos años (Mayol, 2012; Ruiz-Encina y Boccardo, 2014; PNUD, 2017; Araujo, 2019). Así, las primeras protestas se veían materializadas con un descontento general de la población hacia la desigualdad y la falta de conocimiento, o de empatía, de los grupos de élite respecto a la realidad chilena. Esto potenció una discusión, que acabó por deslegitimar una de las mayores herencias autoritarias: la Constitución de 1980 promulgada durante la dictadura militar de Augusto Pinochet. Las críticas ante la Constitución actual consideraban principalmente su legitimidad, el rol subsidiario del Estado y la protección a la propiedad 
privada y los enclaves autoritarios, elementos que terminan conformando la Constitución de 1980 como uno de los pilares dictatoriales que se mantienen hasta hoy (Fundación Sol, 2013).

Desde la esfera política se han realizado diferentes reformas que apuntan a los enclaves autoritarios (Garretón, 2012), así como también el proyecto de una nueva Constitución en el segundo gobierno de Michelle Bachelet, que finalmente no prosperó ni dejó rastros de un posible proceso constituyente. Sin embargo, luego del 18 de octubre ${ }^{4}$ el diálogo sobre una Asamblea Constituyente tomó fuerza en la población, que organizó diversos cabildos abiertos en diferentes comunas del país, con el fin de discutir las problemáticas del país y lo que debiese contener una nueva Constitución.

Finalmente, el 15 de noviembre de 2019, en el ex Congreso Nacional se realiza el Acuerdo por la Paz Social y la Nueva Constitución para comenzar el proceso constituyente. Este acuerdo contemplaba la realización de un plebiscito que consultó a la ciudadanía sobre la aprobación o rechazo de una nueva Constitución y, en caso de aprobarse, el tipo de órgano para redactar la nueva Constitución, es decir, Convención Mixta Constitucional (entre constituyentes elegidos democráticamente y diputados) o Convención Constitucional (solo constituyentes elegidos para la ocasión). A partir de estas opciones se comienzan a configurar los sectores de la sociedad chilena en torno a intereses y expectativas, posibles de plasmar en la nueva Carta Magna. El 25 de octubre de 2020, día que se llevó a cabo el Plebiscito Nacional por una nueva Constitución Política, dejó una categórica decisión: con un 78\% de votantes que optaron por el «Apruebo», el «Rechazo» ganó en la Región Metropolitana solo en Vitacura, Las Condes y Lo Barnechea, comunas que cuentan con los ingresos más altos del país.

Estas cifras han tenido diversos análisis. Por una parte, desde la Coordinadora del Rechazo (El Desconcierto, 2020) se ha considerado que en las comunas donde la opción rechazo tuvo mayor adhesión se encontraría una ciudadanía altamente informada. En cambio, otros diagnósticos plantean que las comunas que representan a la élite económica se encuentran en una desconexión total con la realidad del país y su interés radica en seguir perpetuando sus beneficios socioeconómicos en términos estructurales. Estas declaraciones, y la preliminar observación

4 Fecha en que se data el inicio de las protestas populares que darán comienzo al llamado "estallido social chileno». Estas empiezan con evasiones masivas al Metro de Santiago (transporte público), pero derivarán en manifestaciones masivas que tendrán como foco central la ocupación de la plaza Italia de la capital. 
de los resultados de la votación (al diferenciar tipos de comunas en la región), podría estar dando cuenta de un fenómeno "algo olvidado», «dado por muerto» o que «estuvo perdido» (Pérez-Ahumada, 2013) desde la transición a la democracia en los años 90: la lucha de clases o la conciencia del conflicto de clases.

El presente artículo busca indagar la relación entre posiciones de clase y la votación del Plebiscito Nacional de 2020; cuestión abordada por otras investigaciones en el contexto del estallido social (ArancibiaBustos, 2021). Por medio de un análisis del coeficiente de correlación de Pearson, se pretende dar cuenta de la existencia de una relación, y la fuerza de esta, entre posición de clase y la tendencia a votar «Apruebo» 0 «Rechazo» en el plebiscito, el tipo de órgano y la participación electoral. Este análisis se realizará a nivel comunal, con la información entregada por el Servicio Electoral de Chile (SERVEL) sobre los porcentajes de voto de «Apruebo»y «Rechazo» respecto a una nueva Constitución, el tipo de órgano y la diferencia en la participación de las últimas dos elecciones (el plebiscito y las elecciones presidenciales de 2017). Por otro lado, para la información sobre posiciones de clase en las comunas, se utilizará la Encuesta de Caracterización Socioeconómica Nacional (CASEN) de 2017, aplicando la tipología de las posiciones de clase del sociólogo marxista Erik Olin Wright, en su teoría sobre las explotaciones múltiples.

A modo de hipótesis del presente artículo, se pondrá a prueba la correlación entre posición de clase y la votación del Plebiscito 2020. Específicamente, al considerar la idea de conciencia de clase (y la votación como una forma manifestada del interés material de clase), se sostiene el supuesto de que los votantes del «Apruebo» apuestan por el cambio estructural socioeconómico del país, que sistemáticamente ha favorecido a la burguesía en desmedro de la clase trabajadora; por lo tanto, existirá mayor tendencia en la clase trabajadora a votar «Apruebo» para erradicar, de forma estructural, situaciones de desigualdad. Por otro lado, las clases más altas del país tendrían la tendencia de votar "Rechazo» a modo de seguir perpetuando las ventajas que les trae el actual modelo socioeconómico. Respecto al tipo de órgano que debiese redactar la nueva Carta Magna, se considera que la opción por una Convención Mixta puede implicar un obstáculo para conseguir cambios estructurales, ya que esta alternativa mantendría parte del Congreso en su composición (órgano que se ha visto duramente cuestionado en las protestas de octubre de 2019); en contraste de la opción Convención Constitucional, en la que todos sus participantes son electos en elección popular. Al considerarse la primera opción como un «obstáculo», se considera que esta obtendría mayor apoyo en las posiciones de clase cercanas a la burguesía; en tanto 
la Convención Constitucional obtendría mayores apoyos en las posiciones de clase trabajadora. Por último, sostenemos que mayores niveles de participación pueden identificarse en las posiciones de clase trabajadora, ya que la posibilidad de cambiar la Constitución puede ser vista como una alternativa para reducir los niveles de desigualdad percibida en la sociedad chilena.

La expresión «Del estallido a la lucha de clases» es una puesta, por interpretar el descontento ciudadano por la desigualdad existente. Parte de la literatura ha tendido a interpretar el conflicto social, reflejado en los diferentes movimientos sociales acontecidos desde 2006 en el país, como una expresión del la insatisfacción de los jóvenes de capas medias con el acceso al consumo y el bienestar en el país; incluso, conceptualmente como una pulsión (Peña, 2020). Esta interpretación se basa en investigaciones que plantean el crecimiento de las clases medias cómo un proceso sostenido durante las últimas décadas. En general, se considera que el crecimiento del sector servicios en la estructura productiva del país, junto con el acceso de mayor educación superior (León y Martínez, 2007), contribuye a un fenómeno denominado como mesocratización de la sociedad (Ruiz-Encina y Boccardo, 2014), o el crecimiento de las capas medias. Esta es la base para la conexión entre cambios en la estructura de clase y nuevas formas de conciencia asociadas a esta (conocida como la tesis del malestar). Evidencia reciente pone en cuestión la idea de una mesocratización de la sociedad (Pérez-Ahumada y Cifuentes, 2019), y plantea que niveles altos de desigualdad van acompañados de nuevas formas de ser clase trabajadora. Por tanto, la hipótesis de trabajo se posiciona en un esfuerzo por entender la movilización social de forma distinta al malestar de las clases medias; en tanto puede representar una polarización del conflicto de clases (Arancibia-Bustos, 2021). Estallido, descontento y malestar, por tanto, son formas de invisibilizar la base material del conflicto en el Chile desigual.

En definitiva, lo que se intenta es generar un esfuerzo por volver a relacionar las condiciones de clase (objetivas) con la percepción del conflicto de clase (subjetividad). ¿Existe una relación significativa entre la posición de clase y el posicionamiento político en el actual proceso constituyente? ¿En qué medida la actitud hacia el cambio estructural de la sociedad chilena puede estar determinado condiciones materiales de existencia? ¿Estamos en presencia de un contexto sociopolítico donde cobra relevancia la conciencia subjetiva respecto a las condiciones objetivas? Son parte de las preguntas que intentaremos responder; en un contexto donde la literatura, posterior a la caída de la Unión Soviética, declaró la extinción del proletariado como sujeto político (Gorz, 2001), 
la irrelevancia de la contradicción entre capital y trabajo como factor explicativo de lo social (Bauman, 2000), y el fin de las clases sociales (Pakulski y Waters, 2008).

\section{Materiales y métodos}

\section{Marco de referencia}

A continuación, se procederá a: (1) describir el enfoque de las explotaciones múltiples de Wright, para luego aplicar su esquema a la información empírica disponible, y (2) argumentar por qué se puede considerar a la posición en torno al cambio constitucional como una muestra del establecimiento de cierta conciencia de clase.

Diferentes enfoques sociológicos incluyen en su aparato conceptual la noción de clase. Dentro de los principales enfoques encontramos las que utilizan el concepto de clase como atributos individuales de los agentes para el análisis de clases y la desigualdad; el enfoque de acaparamientos de oportunidades que tiene como principal exponente a Max Weber y el concepto de oportunidades vitales $y$, por último, el enfoque que centra el concepto de clase como explotación y dominación (Wright, 2018).

Siguiendo lo expuesto por Erik Olin Wright (1993), nos centraremos en el concepto de estructura de clase y su relación entre el nivel macro y micro de su análisis, haciendo énfasis en este último para implementar el enfoque de explotaciones múltiples desarrollado por el autor. El autor construye lo anterior para profundizar y dar cuenta de «las relaciones causales entre la estructura de clases, la formación de clase, la conciencia de clase y la lucha de clases» (Wright, 1993, p. 22). Para adentrarse en el concepto de estructura de clase, el autor toma algunos elementos de lo expuesto por John Roemer (1988 y 1989), con el objetivo de superar ciertos problemas teóricos de lo que anteriormente denominó posiciones de clase contradictorias. De Roemer, Wright utiliza el concepto de explotación, que vincula la distribución de los derechos de propiedad, explotación y clase; Roemer da un vuelco hacia una noción de explotación que se aleja de la teoría del valor-trabajo y se asocia más a la idea de las transferencias de trabajo. Junto con lo anterior, el enfoque de la teoría de juegos elaborado por Roemer permite a Wright reconceptualizar su propuesta teórica. Este enfoque «consiste en comparar diferentes sistemas de explotación tratando la organización de la producción como si fuera un juego» (Wright, 1994, p. 74) en el que los jugadores poseen distintos recursos como capital y cualificaciones, con el objetivo de verificar si existe o no explotación y entregar un diagnóstico de las desigualdades económicas en términos de explotación. 
Para el análisis más concreto de la estructura de clase del capitalismo contemporáneo, el autor construye una tipología esquemática de las posiciones de clase del capitalismo (Wright, 1994 y 2018); a saber:

FIGURA 1. Esquema de las explotaciones múltiples para las posiciones de clase según Erik Olin Wright

\begin{tabular}{|c|c|c|c|c|c|}
\hline \multirow[b]{2}{*}{$\begin{array}{c}\text { Posee capital } \\
\text { suficiente para } \\
\text { contratar obreros y } \\
\text { no trabajar }\end{array}$} & \multirow{2}{*}{$\begin{array}{c}\begin{array}{c}\text { Propietarios } \\
\text { de medios de } \\
\text { producción }\end{array} \\
\text { 1. Burguesía }\end{array}$} & \multicolumn{3}{|c|}{ No propietarios (trabajadores asalariados) } & \multirow{4}{*}{ 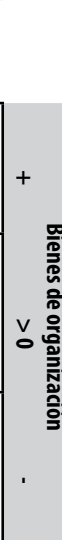 } \\
\hline & & $\begin{array}{l}\text { 4. Experto } \\
\text { directivo }\end{array}$ & $\begin{array}{l}\text { 7. Directivo } \\
\text { semicredencializado }\end{array}$ & $\begin{array}{l}\text { 10. Directivo } \\
\text { no } \\
\text { credencializado }\end{array}$ & \\
\hline $\begin{array}{l}\text { Posee capital } \\
\text { suficiente para } \\
\text { contratar obreros, } \\
\text { pero tiene que } \\
\text { trabajar }\end{array}$ & $\begin{array}{l}\text { 2. Pequeños } \\
\text { empleadores }\end{array}$ & $\begin{array}{l}\text { 5. Experto } \\
\text { supervisor }\end{array}$ & $\begin{array}{l}\text { 8. Supervisor } \\
\text { semicredencializado }\end{array}$ & $\begin{array}{l}\text { 11. Supervisor } \\
\text { no } \\
\text { credencializado }\end{array}$ & \\
\hline $\begin{array}{l}\text { Posee capital } \\
\text { suficiente para } \\
\text { trabajar para sí } \\
\text { mismo, pero no } \\
\text { contrata obreros }\end{array}$ & $\begin{array}{l}\text { 3. Pequeña } \\
\text { burguesía }\end{array}$ & $\begin{array}{l}\text { 6. Experto } \\
\text { no } \\
\text { directivo }\end{array}$ & $\begin{array}{l}\text { 9. Obrero } \\
\text { semicredencializado }\end{array}$ & 12. Proletarios & \\
\hline
\end{tabular}

Nota: esquema de las explotaciones múltiples a partir de Wright (1993), obtenido y elaborado por Pablo Pérez-Ahumada (2007).

A partir de lo descrito en la figura 1, encontramos a los propietarios de los medios de producción y los no propietarios o trabajadores asalariados. Dentro de esta última dimensión, las posiciones de clase se establecen a través de dos relaciones: 1 ) bienes de organización y 2) bienes de cualificación/credenciales. Esta última se delimita a partir de si posee o no posee conocimiento experto amparado por credenciales (donde las instituciones de educación juegan un rol protagónico). Los bienes de organización, en cambio, hacen referencia a las posiciones jerárquicas o mando en la organización del proceso de trabajo (ArancibiaBustos, 2021), al dividir entre quienes planifican y quienes ejecutan el trabajo. Es decir, se diferencia entre gerentes o directores y trabajadores subordinados. De esta manera, expertos directivos, expertos supervisores, expertos no directivos, directivos semicredencializados, supervisores semicredencializados, directivos no credencializados y supervisores no credencializados pueden considerarse como parte de las capas medias, en tanto están atravesadas internamente por condiciones contradictorias de explotación, es decir, posiciones antagónicas; y, en contraste, los obreros semicredencializados y los proletarios se acercan al ideal del proletariado como «clase trabajadora» clásica. Si bien para el caso chileno existen esfuerzos notables por clarificar qué implica ser de clase media, 
tanto a nivel de clasificación (Barozet y Espinoza, 2008; Barozet y Fierro, 2011; Mac-Clure, Barozet, y Maturana, 2014) como de autoidentificación (Espinoza y Barozet, 2008; Castillo, Miranda y Madero-Cabib, 2013; Mac-Clure, Barozet, Galleguillos y Moya, 2015). En la presente discusión presentaremos la noción amplia de sectores medios que, según lo expuesto por Wright, considera a aquellos atravesados por explotaciones múltiples (subordinan, y a la vez son subordinados).

La investigación se estructura como esfuerzo por vincular lo objetivo y lo subjetivo; es decir, la relación entre la clase en sí y la clase para sí (Marx y Engels, 2005). La relación estadística, que se detallará en el siguiente apartado, considera ambos tipos de variables: aquellas que dan cuenta de una estructura de clase (las categorías de Wright), y aquellas que dan cuenta de posicionamiento de clase en sí o conciencia de la clase en particular. Es por esto que dedicaremos un espacio breve para justificar el uso de la votación en torno al cambio constitucional como un aspecto relevante en la construcción de la conciencia de clase.

El estudio de la conciencia de clase en sentido marxista, según PérezAhumada (2013 y 2014), implica conciliar perspectivas estructurales (objetivas) con perspectivas procesuales (subjetivas). De esta última, son característicos en primera instancia el trabajo de Georg Lukács (1970), y en segunda instancia el trabajo del británico Edward Palmer Thompson (1966). La perspectiva procesual plantea como observable empírico la formación de una identidad cultural de clase. En cambio, la perspectiva estructural encabezada por Erik Olin Wright (1997) pone en el centro la idea de interés material de clase. Como propuesta de síntesis de ambas perspectivas, Pérez-Ahumada propone definir conciencia de clase cómo: «el resultado del proceso a través del cual las clases construyen su identidad colectiva y llegan a ser conscientes de sus intereses de clase. Tanto los intereses como la identidad son una creación colectiva de los individuos que se encuentra, sin embargo, estructuralmente constreñida por las relaciones de producción que determinan los límites mismos de la existencia de las clases» (2013, p. 89).

Tomando una de las dimensiones identificadas por Pérez-Ahumada, es posible considerar la intención de voto como un asunto de interés material de clase (a partir de lo esbozado por Wright); más aún cuando la votación va dedicada a un cambio constitucional. Las Constituciones son cartas fundamentales que expresan los valores con los que se conforman los distintos Estados naciones (Atria, 2013); valores cruzados por el consenso político de quienes redactan dicha Carta Magna. El decidir cambiar o no la Carta Magna de un Estado en concreto implica la actitud positiva hacia la modificación de aquellos valores fundantes que reproducen ciertas 
políticas a partir de las leyes del Estado (y el ejercicio de sus poderes). Esta actitud positiva puede tener aparejada una serie de demandas de interés material, expresadas en distintos movimientos sociales (contra las zonas de sacrificio y por el derecho al medioambiente, contra la precariedad laboral, acceso a educación y salud, y fin de las administradoras de fondos previsionales o AFP), los que pueden estar guiando la opinión favorable al cambio de Constitución por parte de la clase trabajadora. Ahora bien, si es posible considerar la intención de voto como un aspecto de interés material de clase, se vuelve necesario ubicar este tipo de manifestación dentro de distintos niveles de desarrollo de la conciencia de clase.

Con aquello en mente, Pérez-Ahumada propone estudiar niveles de desarrollo de la conciencia de clase según lo observado a nivel de literatura (Giddens, 1973; Hazelrigg, 1973; Mann, 1973). Existirían al menos cuatro niveles de desarrollo de la conciencia de clases (Pérez-Ahumada, 2013), enmarcados en: (1) la identidad de clase o formación de la conciencia colectiva subjetiva, (2) oposición de clase o la conciencia de intereses antagónicos de clase, (3) totalidad de clase, o el reconocimiento de cómo los elementos anteriores definen la posición de los sujetos a nivel de sociedad, y (4) la concepción de una sociedad alternativa, como proyecto posible de obtener, en el contexto de la lucha de clases. La presencia de estos cuatro niveles, según el autor, permite hablar de un desarrollo de la conciencia de clase revolucionaria.

Los niveles presentados facilitan la descripción del nivel de la conciencia de clases que se pretende poner a prueba en la presente investigación. Al respecto, se considera que la posición respecto al plebiscito por una nueva Constitución es posible de catalogar como un fenómeno que da cuenta de condiciones propias del segundo nivel de la conciencia de clases; o del nivel que reconoce la existencia de una oposición de clase a partir de los intereses antagónicos. En su investigación, Pérez-Ahumada (2013) ya consideraba a la actitud que se tenía respecto a la intervención del Estado en la economía como un indicador de este segundo nivel de la conciencia de clase (un tanto más avanzada que la conformación de la identidad de clase). La idea de actitudes respecto a la intervención del Estado en asuntos económicos puede rastrearse a nivel de literatura en Chile (González y Madariaga, 2018; Atria, Castillo, Maldonado y Ramirez, 2020; Frei, Castillo, Herrera y Suárez, 2020) a partir del concepto de economía moral (Thompson, 2000); pero no se evidencia que esta sea tratada como una forma de conciencia subjetiva de clase, o de forma conjunta a una posición estructural de los sujetos. 


\section{Metodología}

Tal como muestra la tabla 1, el esquema de Wright (2018) fue adaptado a partir de los datos entregados por la encuesta CASEN de 2017. De dicha encuesta se tomaron tres variables fundamentales, las que cruzadas nos otorgaron 8 de las 12 posiciones del esquema ${ }^{5}$. La primera variable utilizada de la encuesta separa asalariados de empleadores ${ }^{6}$, la segunda directores de no directores ${ }^{7}$, y la tercera nivel educacional ${ }^{8}$. La encuesta CASEN es representativa a nivel nacional y regional, pero no cumple dichas cuotas a nivel comunal (principalmente en las comunas con menor población del país); para solucionar dicho problema, se procedió a seleccionar solo aquellas comunas que cumplían con una cantidad de $\mathrm{n}$ igual o superior al cálculo de su muestreo aleatorio simple (que en la mayoría de los casos oscilaba entre los 300 y 400 casos). Por tanto, se contabilizaron solo a los casos de la muestra que pertenecían a comunas que cumplieron dicha cantidad muestral; al pasar de un total de 324 comunas (con 175076 casos) a una muestra seleccionada de 105 comunas (con 125841 casos). En la tabla 1 se presentan los porcentajes generados por posición de clases tanto en la muestra original obtenida de la CASEN 2017, como en la selección representativa que fue determinada. Es posible notar que las diferencias de puntos porcentuales, para cada posición de clase, no presentan una alta variación entre muestreos; al respecto, la mayor variación con menos de un $3 \%$ se encuentra en la categoría proletarios.

Luego de obtener la posición de clase para cada caso de la muestra, se procede a calcular la proporción que corresponde a cada comuna según cada posición de clase; esto permite ver las variaciones de cada posición de clase según comuna (identificando, por ejemplo, aquellas que tienen mayor presencia de burguesía y aquellas que no). Luego, se cruzó esta

5 Quedan fuera aquellas posiciones donde existen indicadores intermedios de control de los procesos de trabajo. Esto, en la práctica, implica determinar el grado con que se supervisa la producción en las capas medias. En cambio, solo se pudo separar a quienes ostentan cargos directivos o de gerencia en contraste de quienes no, lo que implica una pérdida respecto al nivel intermedio de cadena de mando en la producción. Esto representa una limitación del uso del esquema de Wright con la información disponible en encuestas con representatividad nacional en el caso de Chile.

6 Pregunta «015. En su trabajo o negocio principal, ¿usted trabaja como?»; recodificación categorías de respuesta: «Patrón o empleador». Esto se hace para separar entre dos grupos: burgueses y trabajadores.

7 Pregunta «09.a. ¿Cuál es su ocupación u oficio?»; recodificación categorías de respuesta según la norma ISCO/CIUO 88, dígitos «1»: «Jefes [...]», «Dirigentes [...]», «Director [...]» y «Gerente [...]». Esto se hace para diferenciar directivos y no directivos.

8 Pregunta «e6.a. ¿Cuál es el nivel más alto alcanzado o el nivel educacional actual?»; recodificación categorías de respuesta: «Profesional incompleto (carreras 4 o más años)», «Profesional completo (carreras 4 o más años)», "Posgrado incompleto», "Posgrado completo». Esto se hace para diferenciar tres categorías: expertos, semicredencializados y no credencializados. 
TABLA 1. Diferencia entre muestra seleccionada y muestra original

\begin{tabular}{lccccc}
\hline Variables & \multicolumn{2}{c}{ n seleccionado } & \multicolumn{2}{c}{ n CASEN } \\
& $\mathbf{f}$ & $\%$ & f & $\%$ \\
\hline Burguesía & 840 & $0,67 \%$ & 1004 & $0,57 \%$ \\
\hline Pequeña y mediana burguesía & 890 & $0,71 \%$ & 1195 & $0,68 \%$ \\
\hline Experto directivo & 819 & $0,65 \%$ & 929 & $0,53 \%$ \\
\hline Experto no directivo & 14878 & $11,82 \%$ & 17350 & $9,91 \%$ \\
\hline Directivo semicredencializado & 190 & $0,15 \%$ & 240 & $0,14 \%$ \\
\hline Obrero semicredencializado & 7944 & $6,31 \%$ & 10068 & $5,75 \%$ \\
\hline Directivo no credencializado & 1549 & $1,23 \%$ & 2164 & $1,24 \%$ \\
\hline Proletarios & 98731 & $78,46 \%$ & 142126 & $81,18 \%$ \\
\hline Total general & 125841 & $100 \%$ & 175076 & $100 \%$ \\
\hline
\end{tabular}

Nota: elaboración propia a partir de información extraída de la CASEN 2017.

información con los registros del SERVEL para obtener el promedio por comuna de los siguientes resultados: (1) porcentaje de votación para la opción «Apruebo», (2) porcentaje de votación para la opción «Convención Constitucional», y (3) diferencia porcentual de participación entre la votación del plebiscito de 2020 y la segunda vuelta presidencial de 2017. Luego de obtener dichos porcentajes, se procede a calcular el coeficiente de correlación de Pearson entre las distintas posiciones de clase y los resultados de la votación de Plebiscito Nacional de 2020; esto pues se cumple con el criterio de que las variables presenten un nivel de medición cuantitativo de razón (expresado en porcentajes y/o decimales).

\section{Resultados}

Para el análisis de los resultados se consideran los criterios esbozados por Cohen (1988) en la interpretación de la fuerza de la relación entre variables. Estos criterios plantean que coeficientes entre 0,1 y 0,3 dan cuenta de una fuerza de relación baja, entre 0,3 y 0,5 media, y mayor a 0,5 una fuerza de relación alta.

En la tabla 2, los coeficientes expuestos se aplican a tres variables: «Apruebo», «Convención Constitucional» y «Participación». En primer lugar, en los resultados relativo a la opción «Apruebo» destaca la alta relación negativa que tiene con la burguesía $(-0,599)$ y expertos tanto directivos $(-0,543)$ como no directivos $(-0,487)$, seguida de una fuerza de relación media en la pequeña y mediana burguesía $(-0,231)$. Estos resultados se tornan relevantes si comparamos la relación que tiene el «Apruebo» con el proletariado, ya que presenta una fuerza de relación 
TABLA 2. Coeficiente de correlación de Pearson, entre posición de clase y opción en el plebiscito de octubre de 2020

\begin{tabular}{lccc}
\hline Posición de clase & Apruebo & Cc & Participación \\
\hline Burguesía & $-0,599^{* *}$ & $-0,636^{* *}$ & $-0,149$ \\
\hline Pequeña y mediana burguesía & $-0,231^{*}$ & $-0,201^{*}$ & $-0,037$ \\
\hline Experto directivo & $-0,543^{* *}$ & $-0,618^{* *}$ & $-0,136$ \\
\hline Experto no directivo & $-0,487^{* *}$ & $-0,482^{* *}$ & $-0,099$ \\
\hline Directivo semicredencializado & $-0,078$ & $-0,147$ & 0,002 \\
\hline Obrero semicredencializado & 0,080 & 0,116 & $0,242^{*}$ \\
\hline Directivo no credencializado & 0,108 & 0,084 & 0,097 \\
\hline Proletarios & $0,448^{* *}$ & $0,447^{* *}$ & 0,045 \\
\hline
\end{tabular}

Nota: elaboración propia a partir de información extraída de la CASEN 2017 y los resultados publicados por el SERVEL. ** Correlación significativa en el nivel 0,01 (bilateral), y* correlación significativa en el nivel 0,05 (bilateral).

media (tendiendo a alta) con un coeficiente de correlación de 0,448. En otras palabras, los resultados dan cuenta que existen tendencias de voto en las diferentes posiciones de clases: por un lado, las comunas donde la burguesía presenta una mayor proporción se mantiene un bajo nivel de apoyo a la opción «Apruebo»; y ocurre lo contrario en el caso de las comunas donde el proletariado crece en proporción. Así, se puede dar cuenta de un primer rastro de nuestra hipótesis en torno a los intereses materiales de clases y el segundo nivel de desarrollo de conciencia de clase.

Asimismo, las correlaciones que se establecen entre posición de clase y opción "Apruebo», no varían significativamente en su fuerza de correlación la opción "Convención Constitucional». Por ejemplo, la burguesía presenta un leve aumento en la fuerza de la relación $(-0,636)$, al igual que los expertos directivos $(-0,618)$. En tanto, expertos no directivos $(-0,482)$ y proletariado $(0,447)$ mantienen niveles similares a la opción anterior. Nuevamente, la situación antagónica de clase en la votación del 25 de octubre de 2020 se puede observar en el caso del tipo de órgano en la medida en que la Convención Constituyente supone un cambio de interés que se ve configurado por las posiciones antagónicas de clase.

En materia de participación, las posiciones de clase burguesía, experto directivo y obrero semicredencializado presentan una correlación baja; en tanto, las otras posiciones de clase establecen relaciones no significativas con la variable «Participación». De esta manera, las comunas en las que existe una mayor proporción de posiciones burguesas y de expertos directivos presentan una relación negativa débil en participación con 
respecto a las votaciones de segunda vuelta presidencial celebradas en 2017 en Chile. En cambio, la posición obrero semicredencializado presenta una relación positiva baja en relación con la variable «Participación»; esta sería la posición de clase en la que se puede afirmar cierto aumento en la participación electoral.

Dicho esto, la hipótesis del artículo, como se menciona en apartados anteriores, gira en relación con la correlación existente entre posición de clase y votación en el plebiscito de 2020. Así, podemos dividir la hipótesis en tres partes: 1) Votación por «Apruebo»; 2) Tipo de órgano (Convención Constitucional); y 3) «Participación». Tanto en la parte 1 y 2 de la hipótesis, el proletariado es la posición de clase que mayor correlación presenta respecto a la opción «Apruebo» y el tipo de órgano «Convención Constituyente»; en cambio, las posiciones de clase más altas tienen una correlación negativa tanto con la opción «Apruebo» como con el tipo de órgano (Convención Constituyente). De esta manera, nuestra hipótesis sobre votación y tipo de órgano se cumple en aquellas posiciones más polarizadas del esquema de Wright (burguesía y proletariado). A su vez, se observan falta de pruebas estadísticas para caracterizar a las posiciones medias del esquema, tales como los obreros semicredencializados y los directivos no credencializados. Esto último no ocurre con las posiciones medias-altas que contienen a los grupos profesionales (los expertos), los cuales se posicionan más cercano al interés material de la burguesía que del proletariado.

Sin embargo, respecto a la tercera parte de la hipótesis, donde afirmábamos que la posición de clase proletaria obtendría un mayor porcentaje participación en comparación con el resto de las posiciones de clases, no puede ser aceptada del todo; dado que los resultados arrojan relaciones bajas entre la posición de clase y la participación electoral, en torno al plebiscito y la segunda vuelta presidencial de 2017. Es decir, la tendencia a participar, o no, en las votaciones no se ve determinada por la clase social como variable.

\section{Discusión}

Siguiendo lo expuesto por Pérez-Ahumada (2013 y 2014), en materia de conciencia de clase, hemos propuesto que el voto en el plebiscito de octubre de 2020 se puede interpretar como expresión de la oposición de clase a partir de intereses antagónicos. Posiciones de clase altas, como la burguesía, expertos directivos y expertos no directivos, presentan correlaciones medias-altas negativas con la opción «Apruebo» y "Convención Constituyente»; mientras que la posición proletaria presenta correlaciones medias-altas positivas con las mismas opciones. Esta 
relación no se presenta cuando tenemos en consideración el aumento de participación electoral.

De esta manera, el interés de clase proletario se expresa en la disputa por el contenido de lo deseable para los cambios estructurales, que difieren, en parte, de los intereses de las clases más altas. Así, cambios en materias estructurales implican cambios en las maneras en las que el Estado y el mercado se coordinan y comunican entre sí. En otras palabras, el cambio estructural se puede asociar a la manera en que se desarrollan las múltiples regulaciones en materia de seguridad social, educación, pensiones, salud, medioambiente, trabajo, etc. Entonces, en procesos históricos como el que actualmente vive Chile, encontramos que la posición de la clase proletaria pasa de la clase en sí a la clase para sí, en tanto reconoce sus intereses como antagónicos con las clases más altas (dejando un terreno fértil para la constitución de un proyecto y horizonte de sociedad propio). Esto, de la misma forma, y con un poco más de fuerza en la relación, ocurre con la burguesía; posición de clase que tiene su proyecto ideológico claro (la herencia de la dictadura) y se volcará a defenderlo en las instancias constitucionales.

En un contexto de cuestionamiento a la tesis de la mesocratización de la sociedad (Ruiz-Encina y Boccardo, 2014; Pérez-Ahumada y Cifuentes, 2019), ya expuesta en un comienzo, la presente investigación aporta evidencia relevante para el debate: no solo los sectores medios de la estructura social emergen como un grupo pequeño en comparación con el proletariado, sino que también son estos grupos los que no presentan una relación significativa con las opciones de voto en el plebiscito. Lo anterior, con la excepción del grupo de profesionales expertos (universitarios), los cuales, sobre todo en su variante directiva, o de posiciones gerenciales, parecen reconocerse más con la conciencia de clase burguesa que con la de clase trabajadora (bien alejados de las consignas levantadas desde el «estallido social»). Esta temática, abierta por la posición de los expertos directivos, deja una arista relevante de investigar en términos de conciencia de clase; ya que, pese a pertenecer al grupo trabajador, y al mismo tiempo estar en lo más alto de su jerarquía, este grupo prefiere reconocerse (o aliarse) con la burguesía en términos de clase. ¿Cuáles son las ventajas en términos de interés que ofrece la Constitución neoliberal para los expertos directivos en Chile? ¿Qué otros motivos explican esta «aparente» contradicción en términos de conciencia? Son parte de las preguntas que emergen a partir de estos resultados.

La relación poco significativa de las otras posiciones medias, del esquema de Wright, no solo aporta a la discusión sobre la mesocratización de la sociedad (discusión sobre la estructural social de clases); sino que es 
un hallazgo para la discusión sobre el carácter del conflicto en la sociedad chilena actual. Aparejado a los discursos estructurales sobre el aumento de las capas medias, algunos autores han afirmado la existencia de un malestar (Brunner, 1998; Peña, 2020) como fundante en los conflictos sociales que ha vivido la sociedad chilena de posdictadura. Esta tesis, nacida de las consecuencias directas del periodo autoritario y transición pactada que vivió el país durante los años 90 , refleja un malestar con el proceso de modernización (PNUD, 1998; Lechner, 2006); diagnóstico que en la literatura se sigue sosteniendo casi 20 años después (Peña, 2020). Un malestar provocado por una falta de integración al consumo, las políticas sociales subsidiarias, y una promesa de movilidad social que se ve nublada por el cuestionamiento al valor meritocrático predominante en el Chile neoliberal.

Esta investigación debate y ofrece una nueva perspectiva para interpretar el conflicto social en Chile ( $y$, en particular, el estallido social de 2019). Una perspectiva donde el malestar, además de no tener sustento real en la estructura de clases, parece no explicar la ausencia de una conciencia de la clase media: si la clase media y su proliferación en Chile es una realidad, ¿por qué no emergen en términos de conciencia definida por sus intereses materiales? Desde un enfoque marxista, las clases sociales se reconocen en el conflicto: esto quiere decir que no es posible afirmar que existen solo en términos de estructura social. Los enfoques de estratificación establecen estratos medios a partir de criterios estadísticos definidos por cohortes de porcentaje; sin embargo, solo sirven para afirmar la existencia de estratos y no clases.

Las clases sociales necesitan de dos elementos fundamentales: su posición (en sí) y su reconocimiento (para sí); el conflicto no se puede dejar fuera. Por esto el estudio de la conciencia de clase es relevante para complementar programas de investigación en desigualdades o estratificación: sin este elemento, estamos ordenando la sociedad a nuestra voluntad y con base en nuestros criterios matemáticos, pero no con base en una coherencia interna. Esa coherencia interna está dada por la definición de intereses de clase, pero además por la diferencia cualitativa que existe entre las distintas categorías de los esquemas. Wright ofrece una diferenciación cualitativa significativa entre las distintas categorías de su esquema, basado en intereses teóricos-coherentes que pueden, o no, estar presentes en la sociedad. Precisamente, cuando están ausentes es que podemos hablar de niveles bajos de desarrollo de una conciencia de clase, conflictividad o lucha de clases.

La mesocratización de la sociedad parece difícil de sostener con un proletariado que representa casi el $80 \%$ de la población. El malestar 
de las clases medias no es viable si observamos los resultados de la investigación: polarización clara de resultados en los extremos de la estructura social, y relaciones no significativas desde la posición experto no directivo, hasta directivo no credencializado (las categorías medias del esquema). Tendencias hacia la polarización de las posiciones en términos de intereses de clase, que se suman a su correlación con categorías en específico, dan cuenta de un proceso de antagonismo creciente que puede ser el antecedente para comenzar a hablar de agudización de las contradicciones en el Chile neoliberal. Aún falta algo clave: el contenido de la discusión constitucional; pues la simple voluntad de cambio es una clara limitante para ser taxativo con el juicio emitido en estas conclusiones. Pese a lo anterior, la presente investigación permite retomar esta tesis y ponerla a prueba a medida que pasemos del plebiscito a los resultados de la discusión constitucional en Chile.

¿Cuál es el proyecto de economía-moral que el proletariado apoya, más allá de la intención de cambio en la sociedad chilena? Es la pregunta clave que nace, de cara a las discusiones constitucionales. Todo en un contexto de auge de las candidaturas independientes y la división al interior de los sectores de «izquierda». Esta es una de las barreras de paso entre el segundo nivel de desarrollo de la conciencia de clases y el tercero, ya que, si bien existe intención de cambio, poco se puede decir, aún, respecto a la conformación de un proyecto con intereses de clases para la sociedad chilena, por y para el proletariado.

\section{Contribución de autoría}

Álvaro Arancibia-Bustos, José Flores-Capriles y Susana GutiérrezPeñaloza han participado en la elaboración, el análisis de los resultados, la redacción y el consentimiento de la versión final del presente artículo.

\section{Fuente de financiamiento}

Autofinanciado.

\section{Potenciales conflictos de interés}

Ninguno. 


\section{REFERENCIAS BIBLIOGRÁFICAS}

Arancibia-Bustos, Á. (2021). ¿Malestar de las «clases medias» o lucha de clase? Aportes para una explicación del estallido social chileno. Izquierdas, (50), pp. 1-17.

Araujo, K. (2019). Hilos tensados. Para leer el Octubre chileno. Santiago: Universidad de Santiago de Chile.

Atria, F. (2013). La Constitución tramposa. Santiago: LOM.

Atria, J., Castillo, J., Maldonado, L. y Ramirez, S. (2020). Economic elites' attitudes toward meritocracy in Chile: Toward meritocracy in Chile. American Behavioral Scientist, 64(9), pp. 1219-1241.

Barozet, E. y Espinoza, V. (2008). ¿Quiénes pertenecen a la clase media en Chile? Una aproximación metodológica. Ecuador Debate, (74), pp. 103-121.

Barozet, E. y Fierro, J. (2011). Clase media en Chile, 1990-2011: algunas implicancias sociales y políticas. Santiago: Series de estudios, Fundación Konrad-Adenauer-Stiftung.

Bauman, Z. (2000). Trabajo, consumismo y nuevos pobres. Barcelona: Gedisa.

Brunner, J. J. (1998). Malestar en la sociedad chilena: ¿De qué, exactamente, estamos hablando? Estudios Públicos, 72, pp. 173-19.

Castillo, J. C., Miranda, D. y Madero-Cabib, I. (2013). Todos somos de clase media: Sobre el estatus social subjetivo en Chile. Latin American Research Review, 48(1), pp. 155-173.

Cohen, J. (1988). Statistical power analysis for the behavioral sciences. New York: Routledge.

El Desconcierto. (26 de octubre de 2020). Coordinadora del Rechazo asegura que la opción ganó en Las Condes, Vitacura y Lo Barnechea porque son comunas «altamente informadas». Santiago, Chile.

Espinoza, V. y Barozet, E. (2008). ¿De qué hablamos cuando decimos «clase media»? Perspectivas sobre el caso chileno. Recuperado de http://www2. facso.uchile.cl/sociologia/1060225/docs/clase_media_ex.pdf

Frei, R., Castillo, J. C., Herrera, R. y Suárez, J. I. (2020). ¿Fruto del esfuerzo? Los cambios en las atribuciones sobre pobreza y riqueza en Chile entre 1996 y 2015. Latin American Research Review, 55(3), pp. 477-495.

Fundación Sol. (2013). La violencia estructural y cotidiana a 40 años del golpe: Los 11 pilares dictatoriales que sostienen el actual modelo. Recuperado de https://fundacionsol.cl/blog/actualidad-1/post/laviolencia-estructural-y-cotidiana-a-40-anos-del-golpe-los-11-pilaresdictatoriales-que-sostienen-el-actual-modelo-5662\#o_wblog_post_ main 
Garretón, M. A. (2012). Neoliberalismo corregido y progresismo limitado. Los gobiernos de la Concertación en Chile, 1990-2010. Santiago: Universidad de Arte y Ciencias Sociales (Arcis) y Consejo Latinoamericano de Ciencias Sociales (Clacso).

Giddens, A. (1973). The class structure of the advanced societies. New York: Harper \& Row, Barnes \& Noble.

González, F. y Madariaga, A. (2018). La Constitución social, política y moral de la economía chilena. Santiago: RIL Editores.

Gorz, A. (2001). Adiós al proletariado. Más allá del socialismo. Barcelona: El Viejo Topo.

Hazelrigg, L. E. (1973). Aspects of the measurement of class consciousness. En M. Armer y A. D. Grimshaw, Comparative social research. Methodological problems and strategies (pp. 219-247). New York: John Wiley \& Sons.

Lechner, N. (2006). Obras escogidas. Volumen I. Santiago: LOM.

León, A. y Martínez, J. (2007). La estratificación social chilena hacia fines del siglo XX. En R. Franco, J. Martínez y R. Atria, Estratificación y movilidad social en América Latina. Transformaciones estructurales de un cuarto de siglo (pp. 308-338). Santiago: LOM, Comisión Económica para América Latina y el Caribe (Cepal) y Sociedad Alemana para la Cooperación Internacional (GTZ).

Lukács, G. (1970). Historia y conciencia de clase. Estudios de dialéctica marxista. La Habana: Editorial de Ciencias Sociales del Instituto del Libro.

Mac-Clure, O., Barozet, E. y Maturana, V. (2014). Desigualdad, clase media y territorio en Chile: ¿clase media global o múltiples mesocracias según territorios? EURE, 40(121).

Mac-Clure, O., Barozet, E., Galleguillos, C. y Moya, C. (2015). La clase media clasifica a las personas en la sociedad: Resultados de una investigación empírica basada en juegos. Psicoperspectivas. Individuo y Sociedad, 14(2), pp. 4-15.

Mann, M. (1973). Consciousness and action among the Western working class. London: The Macmillan Press.

Marx, K. y Engels, F. (2005). La ideología alemana. Madrid: Ediciones Akal.

Mayol, A. (2012). El derrumbe del modelo. La crisis de la economía de mercado en el Chile contemporáneo. Santiago: LOM.

Pakulski, J. y Waters, M. (2008). The death of class. En D. Grusky, Social stratification: class, race, and gender in sociological perspective (pp. 10221030). Boulder: Westview Press.

Peña, C. (2020). Pensar el malestar. La crisis de octubre y la cuestión constitucional. Santiago: Taurus. 
Pérez-Ahumada, P. (2007). Explotación y clases sociales. Reflexiones sobre marxismo analítico. Nemesis, (6), pp. 87-101.

Pérez-Ahumada, P. (2013). Encontrando lo que nunca estuvo perdido. Conciencia de clase y conflicto de clases en el régimen neoliberal chileno. Revista de Sociología, (28), pp. 83-111.

Pérez-Ahumada, P. (2014). Cómo entender y estudiar la conciencia de clase en la sociedad capitalista contemporánea. Una propuesta. Theomai, (29), pp. 121-141.

Pérez-Ahumada, P. y Cifuentes, L. (2019). The service industry, privatesector employment and social class in Chile: New developments from labour process theory. Critical Sociology, pp. 1-19.

PNUD. (1998). Las paradojas de la modernización. Santiago: Programa de las Naciones Unidas para el Desarrollo (PNUD).

PNUD. (2017). Desiguales. Orígenes, cambios y desafíos de la brecha social en Chile. Santiago: Programa de las Naciones Unidas para el Desarrollo (PNUD) y Uqbar Editores.

Roemer, J. (1988). Nuevas direcciones en la teoría marxista de las clases sociales. En J. Roemer, El marxismo: una perspectiva analítica. Ciudad de México: Fondo de Cultura Económica.

Roemer, J. (1989). Valor, explotación y clase. Ciudad de México: Fondo de Cultura Económica.

Ruiz-Encina, C. y Boccardo, G. (2014). Los chilenos bajo el neoliberalismo. Clases y conflicto social. Santiago: Nodo XXI y El Desconcierto.

Thompson, E. P. (1966). The making of the English working class. New York: Vintage Books.

Thompson, E. P. (2000). Costumbres en común. Estudios en la cultura popular tradicional. Barcelona: Crítica.

Wright, E. O. (1993). Reflexionando, una vez más, sobre el concepto de estructura de clases. En J. Carabaña Morales y A. De Francisco, Teorías contemporáneas de las clases sociales (pp. 17-126). Madrid: Pablo Iglesias.

Wright, E. O. (1994). Clases. Ciudad de México: Siglo XXI.

Wright, E. O. (1997). Class counts. Comparative studies in class analysis. Massachusetts: Cambridge University Press.

Wright, E. O. (2018). Comprender las clases sociales. Madrid: Akal. 